\title{
MÖSSBAUER SCATTERING FROM A SUPERCOOLED LIQUID
}

\author{
M. Soltwisch, M. E1wenspoek, and D. Quitmann
}

Institut für Atom- und Festkörperphysik, Freie Universität Berlin, Boltzmannstraße 20, D-1000 Berlin 33, Fed.Rep. Germany

\section{ABSTRACT}

By a coherent Mössbauer-scattering experiment, the dynamical structure factor $s(k, \omega)$ was determined for pure liquid glycerol. As a function of temperature $\left(-30^{\circ} \mathrm{C} \leqslant \mathrm{T} \leqslant 0^{\circ} \mathrm{C}\right)$ and momentum transfer $\left(0.7 \AA^{-1} \leq k \leqslant 4 \AA^{-1}\right)$ the width and intensity of the quasielastic line and the intensity of the inelastic Iine were measured. From the ratios of the intensities the mean-square vibrational amplitude of the molecules was derived while from the linewidth of the quasielastic part we extracted translational and rotational diffusion parameters.

\section{INTRODUCTION}

An experiment to study quasielastic non-resonant scattering of photons was performed, which employs the very high energy resolution of Mössbauer-y-radiation. The first system studied was pure supercooled glycerol, a liquid showing slow translational and rotational diffusion of the molecules. The scattered intensity was registered as a function of temperature $\left(-30^{\circ} \mathrm{C} \leqslant \mathrm{T} \leqslant 0^{\circ} \mathrm{C}\right)$ and momentum transfer $\left(0.7 \AA^{-1} \leqslant k \leqslant 4 \AA^{-1}\right)$. Three experimental parameters were obtained at every $(k, T)$-point:

a) the diffusional broadening of the sharp Mössbauer line (fitted by assuming a Lorentzian energy shape of the quasielastic scattering);

b) the ratio of quasielastic to inelastic intensity;

c) the total intensity.

Except for the energy distribution of the inelastic part one measures the dynamical structure factor $s(k, \omega)$ of the liquid system, a quantity otherwise obtainable only by coherent neutron scattering.

\section{EXPERIMENTAL DETAILS AND LIMITATIONS OF THE METHOD}

A $250 \mathrm{mCi}{ }^{57} \mathrm{Co}(\mathrm{Rh})$-source ${ }^{1}(1 \mathrm{~mm} \times 6 \mathrm{~mm} \times 6 \mu)$, inside a brass and lead collimater, a double walled, temperature regulated scattering chamber in Laue-arrangement (transmission scattering) with a sample thickness of $5 \mathrm{~mm}$, and a collimated detector system with Mössbauer absorber and $\mathrm{Si}(\mathrm{Li})-\mathrm{diode}$ were the main parts of the experimental setup. 
They were mounted on a conventional horizontal x-ray goniometer. Around its vertical axis, source and sample could be rotated independent1y. The scattered intensities were corrected for white background and compton-scattering using pulse height spectra taken at different angles.

Without the Mössbauer absorber the apparatus constitutes an $x$-ray scattering arrangement, where the total intensity scattered at angle $\theta$ is proportional to the static structure factor $S(k)$ with $k=4 \pi \sin \theta / \lambda$ and $\lambda=0.86 \AA$. The wavelength used here allows the observation of the next neighbour correlations in condensed systems at moderate angles; one obtains a broad structure maximum from a liquid or sharp Bragg peaks from a single crystal. The occurrence of well defined maxima at $\theta \neq 0$ is an effect of the coherence of the scattering process. This is different from normal Mössbauer-absorption or -emission experiments as they are normally done in studying properties of condensed systems. There one has individual absorption or emission which are perfectly incoherent processes and one has only one fixed value of $\mathrm{k}, \mathrm{k}=2 \pi / \lambda$. Mössbauer scattering now allows one to study correlation between scatterers. For $1 / \mathrm{k} \approx$ molecular distance the response of the system stems to a great part from the correlated arrangements and motions of the scattering sites, while at large momentum transfers $k$ one again observes only the individual molecules or atoms.

We consider cases where the motions of the molecules can be divided into an oscillatory (spatially bounded) part of energy $\mathrm{k}_{B} \mathrm{~T} \approx 20 \mathrm{meV}$ (phonons) and a spatially unbounded portion (recoilfree but Doppler-shifted scattering due to the velocity distribution of the diffusing molecules) producing an energy change of $\leqslant 10^{-7} \mathrm{eV}$. Mössbauer-scattering distinguishes the scattering events due to both parts and the latter, quasielastic part can be energy-analysed. The ratio between the two contributions is governed by a Debye-Waller-factor $f=\exp \left(-\mathrm{k}^{2}\left\langle\mathrm{x}^{2}\right\rangle\right)$, where $\left\langle\mathrm{x}^{2}\right\rangle$ is the mean square vibrational amplitude of the individual molecules; here the coherence of the scattering has to be taken into account.

The energy range of $\leqslant 10^{-7}$ eV for the quasielastic part restricts the applicability of Mössbauer-scattering in liquids to the highly viscous regime. Glycerol is a standard subject in this field and has often been studied by other methods like NMR, NQR, dielectric relaxation, Ramanscattering etc.

\section{RESULTS}

Fig. 1 shows the line-broadenings $W_{m}$ as a function of the scattering angle at three temperatures. Fig. 2 gives the 
measured fraction $F_{m}$ of quasielastic intensity to total intensity for the same temperatures. Fig. 3 shows the inelastic and the total intensity, Iin and $I_{\text {tot, }}$ respectively for one temperature $\left(-16.5{ }^{\circ} \mathrm{C}\right)$. The structure maximum corresponds to the mean molecular distance of $\sim 5 \AA$. The line drawn for $I_{\text {tot }}$ is from a Percus-Yevick hard sphere calculation with an additional fast and independent vibration of the molecules. From the last assumption follows

$$
I_{\text {tot }}=I_{q}+I_{i n}=f \cdot S(k)+(1-f) \cdot S_{S}(k)
$$

where $S_{S}(k)$ is the self-part of $S(k)$ and $S(k)$ is the static structure factor for $\left\langle x^{2}\right\rangle=0$.

In fig. 2, the experimental values $\mathrm{E}_{\mathrm{m}}=I_{\mathrm{q}} / \mathrm{I}_{\text {tot }}$ and the calculated values $f S(k) /\left(\mathrm{fS}_{\mathrm{d}}(\mathrm{k})+\mathrm{S}_{\mathrm{s}}(\mathrm{k})\right)$ are plotted for the three temperatures (note that $S(k)=S_{S}(k)+S_{d}(k)$ ); the agree-
ment is fairly good.

The $\left\langle x^{2}\right\rangle-v a l u e s$ derived in this way show a strong increase with temperature (larger than in the harmonic approximation for the solid where $\left.\left\langle x^{2}\right\rangle \alpha T\right)$. This behavior was already earlier measured by Champeney ${ }^{2}$.

A model which can describe the quasielastic linewidths given in fig. 1, must include translational as well as rotational diffusion. The latter contributes to the line-

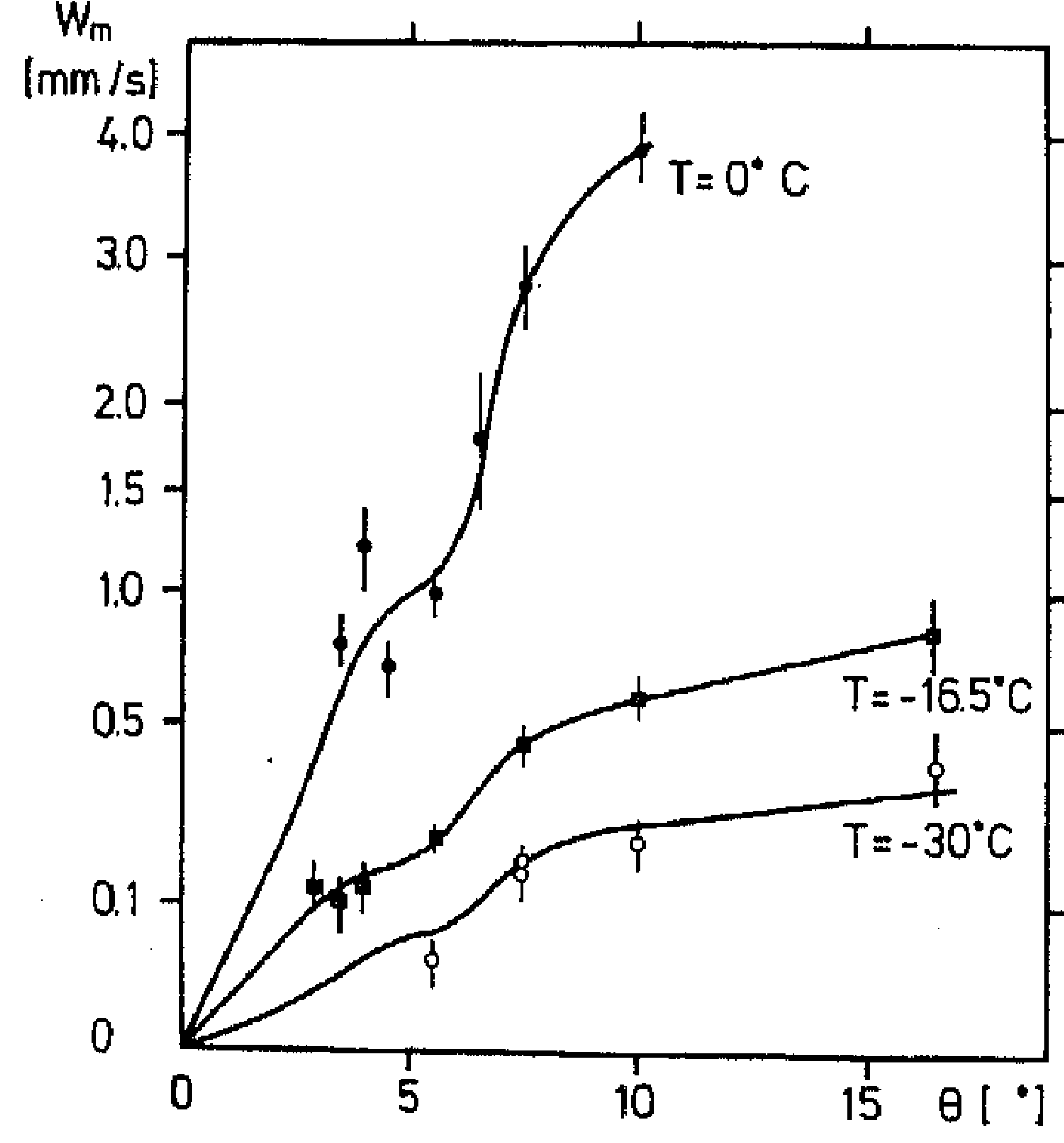
width due to the very unspherical shape of the glycerol molecule. The coherence of the scattering process is essential because it creates a very different k-dependence for the translational and rotational contribution to the linewidths ${ }^{3}$. We assume jump models for both diffusion processes with the same correlation time $\tau$; the mean jump widths are $\left\langle r^{2}\right\rangle$ for the translation and $\left\langle\varepsilon^{2}\right\rangle$ for the rotation. These three quantities are determined from a fit to the linewidth as a function of

Fig. 1. Broadenings of the quasielastic scattered intensities as a function of the scattering angle $\theta, \mathrm{k}=4 \pi \sin \theta / \lambda$. Results: $\left[\tau\right.$ in $\left.10^{-7} \mathrm{~s}\right]$ $T=-30.0^{\circ} \mathrm{C}: \tau=1.0(2),\left\langle r^{2}\right\rangle=0.10 \ldots 1.0 \AA^{2},\left\langle\varepsilon^{2}\right\rangle=0.1 \ldots 1.0 \mathrm{rad}^{2}$ $T=-16.5^{\circ} \mathrm{C}: \tau=0.4(2),\left\langle r^{2}\right\rangle=0.80 \ldots 1.3 \AA^{2},\left\langle\varepsilon^{2}\right\rangle=0.05 \ldots 0.4 \mathrm{rad}^{2}$ $\mathrm{T}=0.0^{\circ} \mathrm{C}: \tau=0.07(2),\left\langle\mathrm{r}^{2}\right\rangle=0.80 \ldots 1.3 \mathrm{~A}^{2},\left\langle\varepsilon^{2}\right\rangle=0.25 \ldots 0.7 \mathrm{rad}^{2}$ 
$k$. These results are given in the capture of fig. 1. From these, the diffusion constants $D_{\text {trans }}=\left\langle r^{2}\right\rangle / \sigma \tau$ and $D_{\text {rot }}=$ $\left\langle\varepsilon^{2}\right\rangle / 6 \tau$ can be derived; their temperature dependence follows an Arrhenius law. The parameters of translational motion can be determined considerably better than the rotational ones. For a more detailed discussion of parts of the results see ${ }^{4}$.

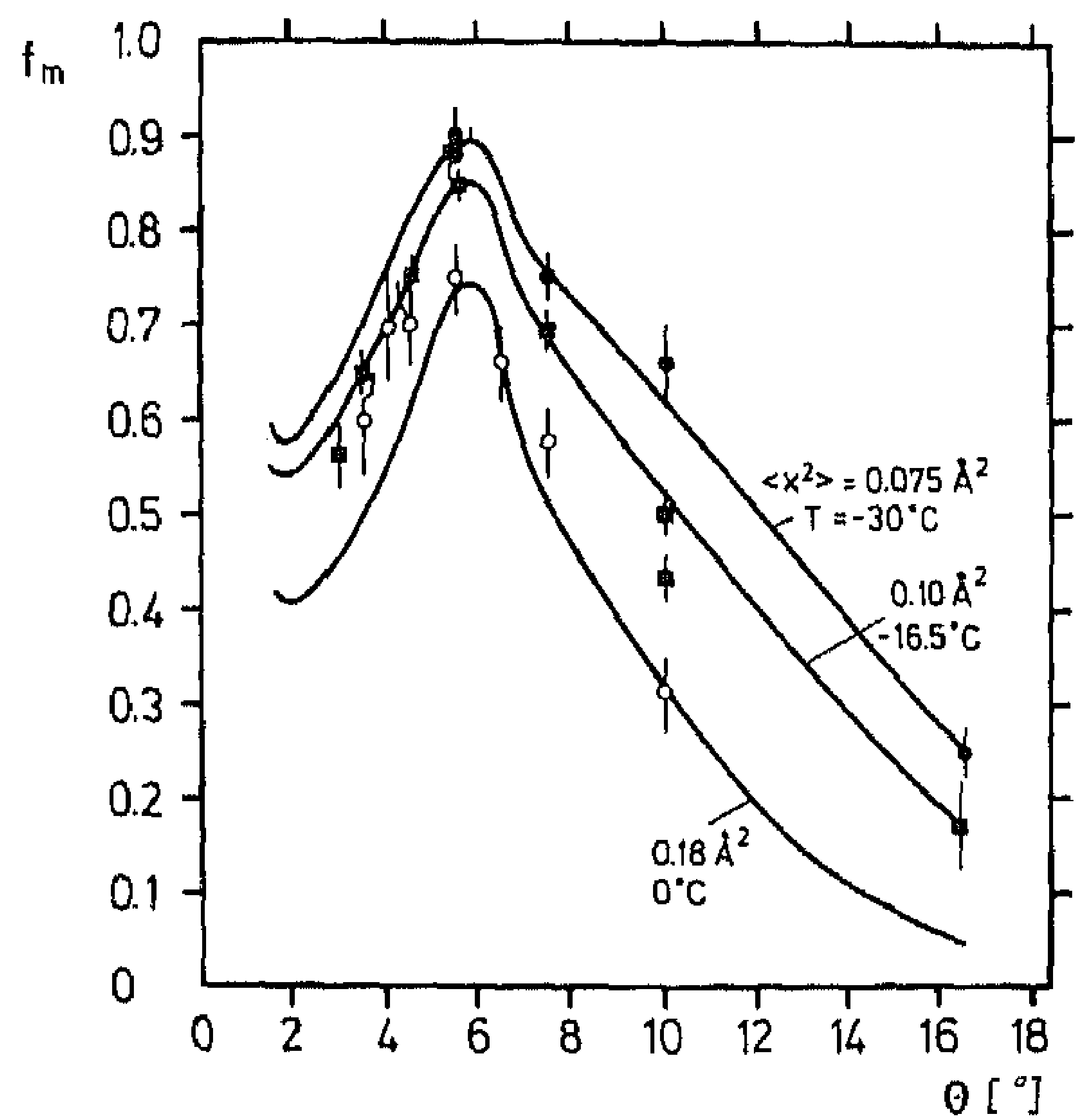

Fig. 2. Ratio of quasielastic to total intensity as a function of scattering angle $\theta$ for three temperatures.

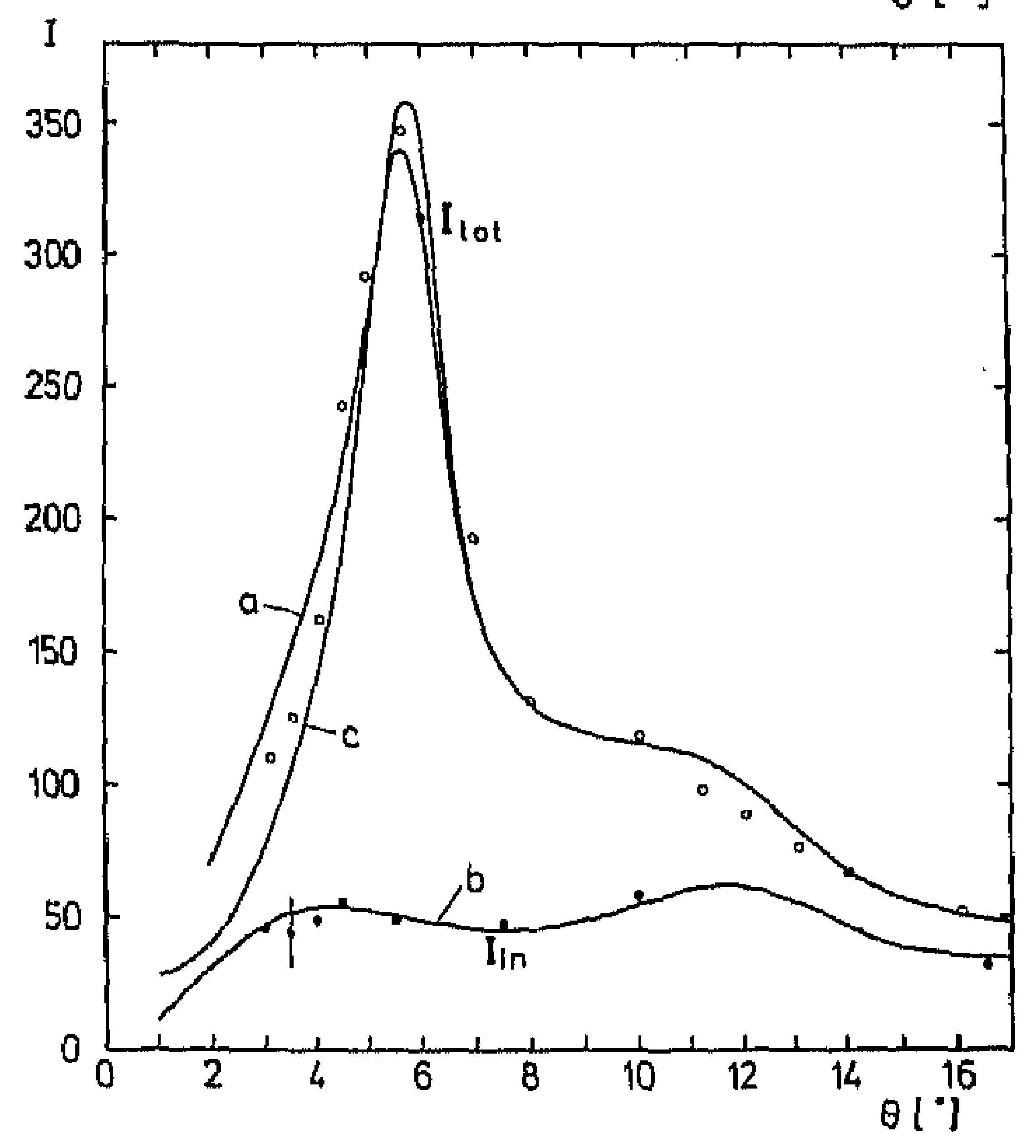

Fig. 3. Total and inelastic scattering intensity $\mathrm{I}_{\text {tot }}=$ $I_{q}+I_{\text {in }}$ and $I_{m}$, respectively. Points: experimental results $\left(\mathrm{T}=-16.5^{\circ} \mathrm{C}\right)$. Curve a gives $I_{\text {tot }}$ and curve $b$ is $I_{i n}$, calculated with $\left\langle\mathrm{x}^{2}\right\rangle=0.1 \AA^{2}$. Curve $c: I_{\text {tot }}=I_{q}$ calculated for $\left\langle x^{2}\right\rangle=0$. The intensities are corrected for background and Compton-scattering.

1. Radiochemical Centre, Amersham, England.

2. Champeney, D.C., and Woodhams, F.W.D., J. Phys. B, 1 , $620(1968)$.

3. Sears, V.S., Can.J.Phys., 44,1299 (1966).

4. Soltwisch, M., Elwenspoek, M., Quitmann, D., appears in Molecular Physics 1977. 